\title{
Azithromycin combined with doxycycline in non-gonococcal urethritis
}

\author{
ZHONGMING LU ${ }^{1 *}$, MANLI HOU ${ }^{2 *}$, DANDAN LI $^{3}$, JING CHENG $^{4}, \mathrm{HUAZHOU} \mathrm{DENG}^{5}$ and JIANPING YAN ${ }^{6}$ \\ ${ }^{1}$ Department of Dermatology, Shengzhou People's Hospital, Shengzhou Branch Hospital of The First Affiliated \\ Hospital of Zhejiang University, Shaoxing, Zhejiang 312400; ${ }^{2}$ Department of Dermatology, Zaozhuang Hospital, \\ Zaozhuang Mining Group, Zaozhuang, Shandong 277100; ${ }^{3}$ Department of Gynecology, Shengzhou People's Hospital, \\ Shengzhou Branch Hospital of The First Affiliated Hospital of Zhejiang University, Shaoxing, Zhejiang 312400; \\ ${ }^{4}$ Department of Dermatology, Shaoxing Shangyu People's Hospital, Shaoxing, Zhejiang 312300; \\ ${ }^{5}$ Department of Doctor-Patient Office, Shengzhou People's Hospital, Shengzhou Branch Hospital of The First \\ Affiliated Hospital of Zhejiang University, Shaoxing, Zhejiang 312400; ${ }^{6}$ Department of Dermatology, \\ Xinchang People's Hospital, Shaoxing, Zhejiang 312500, P.R. China
}

Received September 17, 2019; Accepted May 13, 2020

DOI: $10.3892 /$ etm.2020.9125

\begin{abstract}
Non-gonococcal urethritis (NGU) is one of the most common sexually transmitted diseases caused by chlamydia or mycoplasma. The present study aimed to explore the clinical efficacy of azithromycin combined with doxycycline in patients with NGU and its effect on serum levels of inflammatory cytokine interleukin-6 (IL-6).A total of 98 patients with non-gonococcal urethritis were prospectively selected, of which 46 patients were assigned to an azithromycin group (treated with azithromycin alone), while the rest were assigned to a combination group (treated with azithromycin and doxycycline). The conditions of the patients were evaluated and compared between the two groups. The treatment efficacy in patients in the combination group was significantly better than that in the azithromycin group, and the time to symptom relief, period of medication, recurrence rate within 1 year of withdrawal and level of serum IL-6 in the combination group were significantly lower than those in the azithromycin group. The quality of life of patients in the combination group was significantly improved compared to those in the azithromycin group. However, there was no significant difference in the incidence of adverse reactions between the two groups. Azithromycin combined with doxycycline was revealed to be more effective than azithromycin monotherapy for NGU.
\end{abstract}

Correspondence to: Dr Jianping Yan, Department of Dermatology, Xinchang People's Hospital, 117 Gushan Middle Road, Nanming Street, Xinchang, Shaoxing, Zhejiang 312500, P.R. China

E-mail: tpy8br@163.com

*Contributed equally

Key words: azithromycin, doxycycline, non-gonococcal urethritis, clinical efficacy, IL-6

\section{Introduction}

Non-gonococcal urethritis (NGU) is one of the most common sexually transmitted infections caused by Chlamydia trachomatis, Mycoplasma genitalium, and Neisseria gonorrhoeae (1). Mycoplasma, the pathogen of NGU, can adhere to the surface of the genitourinary tract, resulting in an epithelial infection (2). In recent years, with changes in life-style and sexual behavior of people, the incidence of NGU is increasing, and the primary clinical manifestation is urethritis, generating a serious impact on the quality of life and health of people (3).

At present, patients with NGU are treated with azithromycin (a broad-spectrum drug) monotherapy (4). Azithromycin, a macrolide, is useful to treat infections caused by Gram-positive aerobic bacteria such as Staphylococcus aureus (5). However, due to its widespread clinical application in recent years, several pathogens have developed resistance against azithromycin. Additionally, azithromycin has many adverse reactions (6). Therefore, although it has an effect on NGU, there are still some limitations.

Doxycycline is a long-acting tetracycline-based broadspectrum antibiotic and has a high fat solubility $(7,8)$. Doxycycline prevents protein synthesis by binding to nucleosome $30 \mathrm{~S}$ subunit of pathogenic bacteria, and inhibits the replication of bacterial protein DNA, thus killing the pathogen $(7,8)$. Previous research has revealed that doxycycline has better tolerance, a longer half-life period and a stronger antibacterial activity compared to tetracycline (9). However, due to the wide application of antibiotics in recent years, common pathogenic bacteria achieve certain resistance against antibiotics, leading to unsatisfactory antibiotic monotherapy treatment (10). However, a previous study revealed that Ureaplasma urealyticum and Mycoplasma hominis were highly sensitive to doxycycline, and were not resistant against doxycycline (11). Previous studies have also revealed that multiple antibiotics are more effective than monotherapy in treating NGU (12). Therefore, in the present study, the efficacy 
of azithromycin and doxycycline combination therapy was evaluated for the treatment of NGU.

Interleukin-6 (IL-6) is released by a monocyte in response to a bacterial or viral infection (13). As one of the primary inflammatory factors, IL-6 can reflect the degree of inflammatory response of the immune system against the invading pathogen (13). A previous study revealed that urethral mucosa in patients with non-gonococcal Mycoplasma urethritis promoted IL-6 secretion which in turn increased inflammation (14). It is suggested that IL-6 plays a pivotal role in the inflammatory response towards NGU.

At present, there are relatively few studies on the application of azithromycin and doxycycline combination therapy in NGU and their effects on IL-6. The present study aimed to explore the efficacy of this combination therapy in NGU and the effects of doxycycline on the inflammatory response by assessing the expression of IL-6.

\section{Patients and methods}

General data. The present study was conducted from January 2016 to January 2017, and 98 patients with NGU were prospectively selected, including 47 males and 51 females, with an average age of $35.2 \pm 2.4$ years, and the sample size was estimated as previously described (15). The patients were assigned to an azithromycin group (46 patients, treated with azithromycin alone) or a combination group (52 patients, treated with azithromycin and doxycycline).

Inclusion and exclusion criteria. The inclusion criterion was as follows: Patients diagnosed with NGU by PCR detection of Mycoplasma and chlamydia in urethral discharge during their first visit (16).

The exclusion criteria were as follows: i) Patients with severe organ diseases, such as liver, kidney as well as other organ diseases; ii) patients who had taken antibiotics 2 weeks before being enrolled in the study; iii) patients allergic to azithromycin or doxycycline; iv) patients who had taken doxycycline and azithromycin; v) patients who had taken drugs such as cephalosporins with effects onthe efficacy of doxycycline or azithromycin; vi) patients infected with Neisseria gonorrhoeae or any other STIs; vii) patients withrepeated unprotected sex during the study period; viii) patients who did not receive any physical examination; ix) patients with communication disorder or cognitive disorders.

All patients and their family members agreed to participate in the study and signed informed consent documents. The present study was approved by the Ethics Committee of Xinchang People's Hospital.

Therapeutic methods. Azithromycin dispersible tablets were administrated to patients in the azithromycin group once a day at a dose of $1 \mathrm{mg}, 2 \mathrm{~h}$ after a meal and the dosage was doubled during the first use, and then it was returned to the normal dosage. Patients in the combination group were administered enteric-coated doxycycline hydrochloride tablet at $100 \mathrm{mg}$ once a day along with azithromycin dispersible tablets. The dosage was doubled during the first use, and then it was returned to the normal dosage, and the tablet was taken after meals for a 7-day treatment course. In addition, the patients were closely observed.
The withdrawal time was recorded. The indexes were evaluated after two courses of treatment. Patients who failed to respond to the treatment were provided with another therapy plan.

Observation indicators. Observation indicators were as follows: i) After two weeks of treatment, the therapeutic efficacy of the two groups was evaluated. The therapeutic efficacy was divided into recovery, onset of action, improvement and inefficacy. Efficacy evaluation criteria were as follows (17): a) Recovery, disappearance of symptoms, and negative results in the diagnostic test; b) onset of action, relief in signs and symptoms and negative results in the diagnostic test; c) improvement, remission in signs and symptoms and positive results in the diagnostic test; inefficacy, no improvement in signs and symptoms with lack of relief and even increased severity of infection, with positive results in the diagnostic test. The total efficacy rate was calculated as follows: Total efficacy rate $=$ (Number of recovery cases + number of onset of action cases)/total number of cases $\mathrm{x} 100 \%$. ii) The symptom relief time and medication period ( 2 courses of treatment each consisting of 7 days) of the two groups were recorded and compared. iii) The recurrence in the two groups within one year after treatment was terminated, if any, was recorded and compared. Patients who failed to respond to the treatment were not included in the evaluation. The recurrence criteria were symptom recurrence and positive mycoplasma or chlamydia culture from urethral/cervical secretion. iv) The enzyme-linked immunosorbent assay (ELISA) was used to assess and compare serum IL-6 levels in the two groups of patients before treatment and 1 week after drug withdrawal. ELISA was performed according to the kit instructions (Shanghai Enzyme-Linked Biotechnology Co., Ltd., China). v) Adverse reactions such as headaches, abdominal pain, nausea and vomiting and rash that occurred during the treatment were recorded and compared between the two groups. vi) The Quality of life (QoL) core questionnaire, the QLQ-C30 by EORTC (18), primarily designed for use in cancer clinical trials, but that can be used in other research, was used to evaluate the quality of life in patients of the two groups after 2 courses of treatment. This questionnaire is comprised of 5 function scales (role, emotional, somatic, cognitive and social), with a total of 30 items. A higher score indicated better quality of life and functioning. The results of the QoL questionnaires were analyzed according to the specific scoring manual provided by the EORTC (19), all scores were expressed on a scale ranging between 0-100.

Statistical analysis. The measured data were expressed as the mean \pm standard deviation. The independent t-test was used to compare data between the two groups. Paired t-test was used to compare data before and after the treatment in both groups. Direct counts were expressed as percentages and measured by the $\chi^{2}$ test. Differences were considered significant when $\mathrm{P}<0.05$. All statistical analyses were carried out in SPSS v19.0 (AsiaAnalytics formerly SPSS China).

\section{Results}

Comparison of general data. There were no significant differences in sex and age as well as other factors between the two groups (all $\mathrm{P}>0.05$; Table I). 
Table I. General data (n, \%).

\begin{tabular}{|c|c|c|c|c|}
\hline Factors & Azithromycin group $(\mathrm{n}=46)$ & Combination group $(\mathrm{n}=52)$ & $\mathrm{t} / \chi^{2}$ tests & P-value \\
\hline Sex & & & 0.001 & 0.980 \\
\hline Male & $22(47.83)$ & $25(48.08)$ & & \\
\hline Female & $24(52.17)$ & $27(51.92)$ & & \\
\hline Age (years) & & & 0.002 & 0.960 \\
\hline$\leq 35$ & $21(45.65)$ & $24(46.15)$ & & \\
\hline$>35$ & $25(54.35)$ & $28(53.85)$ & & \\
\hline BMI $\left(\mathrm{kg} / \mathrm{m}^{2}\right)$ & & & 0.027 & 0.869 \\
\hline$\leq 22$ & $24(52.17)$ & $28(53.85)$ & & \\
\hline$>22$ & $22(47.83)$ & $24(46.15)$ & & \\
\hline Marriage & & & 0.010 & 0.922 \\
\hline Yes & $35(76.09)$ & $40(76.92)$ & & \\
\hline No & $11(23.91)$ & $12(23.08)$ & & \\
\hline \multicolumn{5}{|l|}{ Liver function } \\
\hline Serum total protein $(\mathrm{g} / \mathrm{l})$ & $72.31 \pm 2.13$ & $72.34 \pm 2.11$ & 0.070 & 0.944 \\
\hline Alanine aminotransferase $(\mu \mathrm{mol} / \mathrm{l})$ & $28.42 \pm 4.57$ & $27.93 \pm 4.52$ & 0.533 & 0.595 \\
\hline Total bilirubin $(\mu \mathrm{mol} / \mathrm{l})$ & $11.22 \pm 2.12$ & $11.21 \pm 2.04$ & 0.024 & 0.981 \\
\hline \multicolumn{5}{|l|}{ Renal function } \\
\hline Creatinine $(\mu \mathrm{mol} / \mathrm{l})$ & $66.19 \pm 3.05$ & $67.02 \pm 3.12$ & 1.328 & 0.187 \\
\hline Serum urea $(\mathrm{mmol} / \mathrm{l})$ & $5.05 \pm 0.41$ & $5.06 \pm 0.38$ & 0.125 & 0.901 \\
\hline Uric acid $(\mu \mathrm{mol} / \mathrm{l})$ & $269.11 \pm 10.44$ & $271.32 \pm 10.31$ & 1.053 & 0.295 \\
\hline
\end{tabular}

BMI, body mass index.

Comparison of clinical efficacy between the two groups. The total effective rate in the azithromycin group was lower than that in the combination group (52.17 vs. $84.62 \%, \mathrm{P}<0.001$; Table II).

Comparison of symptom relief time and medication time in two groups. The symptom relief time and medication period (2 courses of treatment each consisting of 7 days) in the azithromycin group were $8.12 \pm 1.31$ and $11.42 \pm 2.51$ days, respectively. The same indicators in the combination group were $5.79 \pm 1.14$ and $8.85 \pm 2.11$ days, respectively. The symptom relief time and medication period in the combination group were also significantly lower than in the azithromycin group $(\mathrm{P}<0.001$; Table III).

Recurrence after withdrawal in the two groups. A total of 10 patients in the azithromycin group had a recurrence within one year after the treatment was terminated, with a recurrence rate of $21.74 \%$. In the combination group, 3 patients had a recurrence within one year after withdrawal, with a recurrence rate of $5.77 \%$. The recurrence rate in azithromycin group was significantly greater than the combination group $\left(\chi^{2}=5.410\right.$, $\mathrm{P}=0.020$; Fig. 1).

Serum IL-6 levels in patients before and after treatment in two groups. Serum IL-6 levels in the azithromycin group before and after treatment were $19.73 \pm 4.29$ and $15.36 \pm 2.91 \mathrm{pg} / \mathrm{ml}$, respectively. However, serum IL-6 levels in the combination group before and after treatment were $20.02 \pm 4.14$ and $12.12 \pm 2.05 \mathrm{pg} / \mathrm{ml}$, respectively. There was no significant difference in the serum IL-6 levels between the two groups before treatment $(\mathrm{P}>0.05)$. After treatment, the levels of serum IL-6 in the two groups were significantly lower than those before treatment $(\mathrm{P}<0.05)$ and serum IL-6 in the combination group was significantly lower than that in the azithromycin group $(\mathrm{P}<0.05$; Fig. 2).

Incidence of adverse reactions during treatment in both groups. The occurrence of headaches, abdominal pain, nausea and vomiting, and rash in the azithromycin group and combination group were 1,2,1,2 and 1,2,2,2, respectively, which revealed no intergroup difference in the incidence of adverse reactions (13.04\% vs. $13.46 \%, \mathrm{P}>0.05$; Table IV).

Comparison of the quality of life between the two groups after two courses of treatment. There was no significant difference in the quality of life score between the two groups before treatment $(\mathrm{P}>0.05)$. After treatment, the quality of life score in the two groups was significantly greater than that before treatment $(\mathrm{P}<0.05)$. After treatment, the role, emotional and somatic, cognitive and social functions in the combination group were significantly greater than those in the azithromycin group (all $\mathrm{P}<0.05$; Table V).

\section{Discussion}

NGU is a sexually transmitted disease caused by Chlamydia trachomatis or Mycoplasma hominis. Mycoplasma and chlamydia are common sexually transmitted pathogens in males 
Table II. Clinical efficacy in the two groups (n, \%).

\begin{tabular}{lccc}
\hline Efficacy & Azithromycin group (n=46) & Combination group (n=52) & $\chi^{2}$ test \\
\hline Healing achieved & $14(30.43)$ & $28(39.02)$ & \\
Markedly effective & $10(21.74)$ & $16(31.71)$ & \\
Improvement achieved & $16(34.78)$ & $6(17.07)$ & \\
Ineffective & $6(2.22)$ & $2(12.20)$ & 12.09 \\
Total effective rate & $24(52.17)$ & $44(84.62)$ & $<0.001$ \\
\hline
\end{tabular}

Table III. Time course of symptom relief and medication in the two groups.

\begin{tabular}{lrrr}
\hline Parameter & Azithromycin group (n=46) & Combination group (n=52) & t-value \\
\hline Time course of symptom relief (days) & $8.12 \pm 1.31$ & $5.79 \pm 1.14$ & 9.415 \\
Medication period (days) & $11.42 \pm 2.51$ & $8.85 \pm 2.11$ & $<0.001$ \\
\hline
\end{tabular}

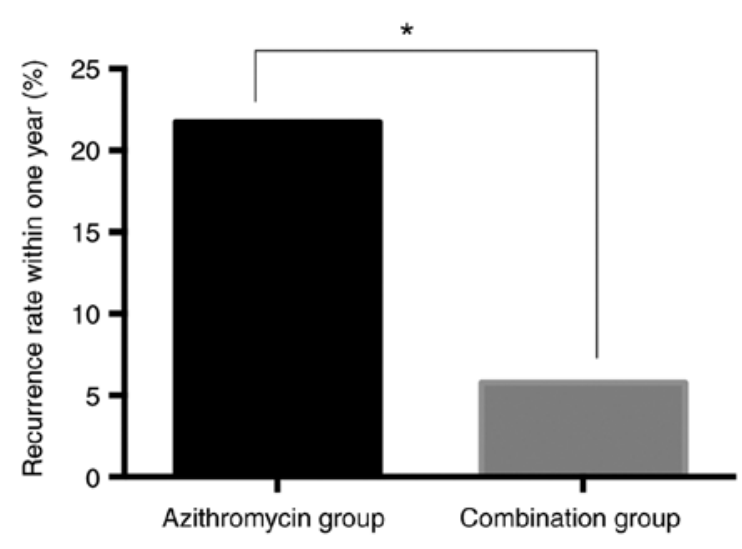

Figure 1. Recurrence within one year after treatment was terminated in the two groups. ${ }^{*} \mathrm{P}<0.05$.

and females, both of which can cause stubborn infections that cannot be cured by antibiotics. The resulting disease from a resistant pathogen is difficult to cure and prone to recurrence $(20,21)$. Since the primary route of transmission of NGU is sexual, the incidence of NGU has increased rapidly in recent years, and it has become primarily a sexually transmitted disease. It generally affects the health of women, but it also has a negative impact on the male reproductive system, and can easily lead to sterility. Hence, it is a threat to the health of people and general well-being $(22,23)$. Drug therapy is the treatment of choice for patients with NGU. However, due to the abuse of antibiotics, drug resistance of pathogens has significantly increased, which renders treatment of the disease difficult. It is, therefore, imperative to find alternative and effective treatment methods for NGU $(24,25)$.

The therapeutic effects of azithromycin monotherapy and azithromycin combined with doxycycline on NGU were compared in the present study. It was revealed that the efficacy of the azithromycin group was significantly lower than that of the combination group. The symptom relief time and medication period in the combination group were also significantly lower than in the azithromycin group.

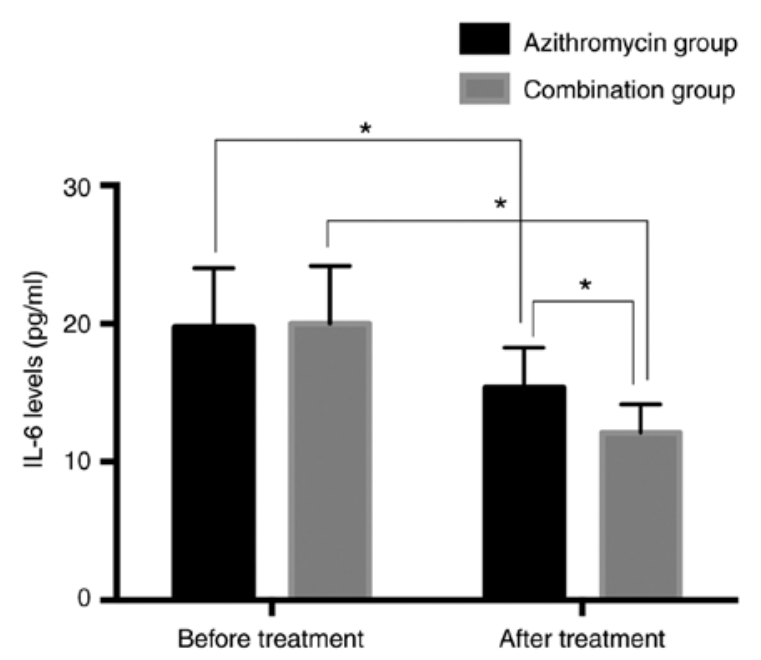

Figure 2. Serum IL-6 levels in patients before and after treatment in the two groups. ${ }^{*} \mathrm{P}<0.05$. IL-6, interleukin- 6 .

Hence, the combination of azithromycin and doxycycline was more effective in the treatment of NGU. Azithromycin is commonly used for the treatment of NGU caused by Mycoplasma infection, and its irregular use has led to mutations in drug resistance genes in Mycoplasma (26). In Denmark, azithromycin resistance in Mycoplasma genitalium isolates have been found to be as high as $40 \%$ (27). Doxycycline is highly effective as an alternative therapeutic drug for NGU caused by Mycoplasma. Mycoplasma is also highly sensitive to doxycycline NGU (26). Although there are relatively few studies on the efficacy of azithromycin in combination with doxycycline in the treatment of NGU, the present study revealed that the combination therapy can be very useful. To further evaluate the effects of the combination therapy, the recurrence rate, adverse reactions and serum IL-6 levels before and after treatment between the two groups were compared. The results revealed that the recurrence rate and serum IL-6 level in the combination group were lower than those in the azithromycin group after one 
Table IV. Incidence of adverse reactions in the two groups (n, \%).

\begin{tabular}{lccc}
\hline Adverse reactions & Azithromycin group (n=46) & Combination group (n=52) & $\chi^{2}$ test \\
\hline Headaches & $1(2.17)$ & $1(1.92)$ & P-value \\
Abdominal pain & $2(4.35)$ & $2(3.85)$ & $2(3.85)$ \\
Nausea and vomiting & $1(2.17)$ & $2(3.85)$ & 0.004 \\
Rash & $2(4.35)$ & $7(13.46)$ & 0.951 \\
Total incidence rate & $6(13.04)$ & \\
\hline
\end{tabular}

Table V. Comparison of the quality of life between the two groups.

\begin{tabular}{lcrr}
\hline Parameters & Azithromycin group $(\mathrm{n}=46)$ & Combination group $(\mathrm{n}=52)$ & t-value \\
\hline Before treatment & & & 0.815 \\
$\quad$ Role function & $56.11 \pm 2.56$ & $55.99 \pm 2.49$ & 0.235 \\
Emotional function & $55.39 \pm 2.61$ & $56.12 \pm 2.72$ & 1.351 \\
Somatic function & $55.73 \pm 2.55$ & $55.59 \pm 2.31$ & 0.285 \\
Cognitive function & $56.02 \pm 2.94$ & $55.83 \pm 2.84$ & 0.325 \\
Social function & $55.17 \pm 2.83$ & $55.26 \pm 2.79$ & 0.158 \\
After treatment & & & 0.746 \\
Role function & $72.67 \pm 2.12^{\mathrm{a}}$ & $80.45 \pm 2.23^{\mathrm{a}}$ & 0.875 \\
Emotional function & $70.82 \pm 2.23^{\mathrm{a}}$ & $79.91 \pm 2.06^{\mathrm{a}}$ & 17.64 \\
Somatic function & $70.99 \pm 2.18^{\mathrm{a}}$ & $81.47 \pm 2.02^{\mathrm{a}}$ & 20.97 \\
Cognitive function & $73.68 \pm 3.02^{\mathrm{a}}$ & $80.33 \pm 2.87^{\mathrm{a}}$ & $<0.001$ \\
Social function & $70.27 \pm 2.16^{\mathrm{a}}$ & $81.52 \pm 2.46^{\mathrm{a}}$ & $<0.001$ \\
\end{tabular}

Compared with before treatment, ${ }^{\mathrm{a}} \mathrm{P}<0.05$.

year after the termination of treatment. Moreover, there was no significant difference in the incidence of adverse reactions between the two groups. This may, thus, indicate that the addition of doxycycline to azithromycin monotherapy can not only reduce the recurrence of NGU of patients, but also alleviate the inflammatory reaction without causing more adverse events. One previous study has revealed that $90 \%$ of doxycycline is absorbed through the gastrointestinal tract when taken orally; in addition to having fewer side effects (28). In addition, doxycycline, a tetracycline, acts as a powerful antibiotic by mainly blocking the protein synthesis of pathogens by binding specifically to ribosomal $30 \mathrm{~S}$ subunits. It has higher fat solubility and a strong tissue penetration ability and also causes fewer side-effects in patients with renal dysfunction $(29,30)$. Previous studies have indicated that doxycycline may be more effective than azithromycin in the treatment of rectal chlamydia infection (31). Although the authors did not evaluate the combination therapy, they concluded that doxycycline was more effective than azithromycin. Finally, the quality of life between the two groups was compared, and it was revealed that the quality of life score of the azithromycin group was significantly lower than that of the combination group. Hence, azithromycin combined with doxycycline was revealed to improve the therapeutic efficacy and the quality of life of patients.
However, there are some limitations in the present study. For example, the impact of different courses of treatment on patients as well as the efficacy of doxycycline monotherapy were not explored. A previous study revealed that $1 \mathrm{~g}$ azithromycin was more effective than doxycycline used for Mycoplasma genitalium in treating nongonococcal urethritis (25), however we have yet to investigate this. In addition, we have not analyzed the effectiveness of IL- 6 level and the correlation of IL- 6 level with clinical efficacy and adverse reactions. These topics may be explored in a future study.

In conclusion, azithromycin combined with doxycycline was revealed to be more effective than azithromycin monotherapy in the treatment of non-gonococcal urethritis. It could effectively alleviate the symptoms of patients and reduce the expression of inflammatory factors such as IL-6 in the serum of patients, improve the quality of life of patients, without causing more adverse reactions.

\section{Acknowledgements}

Not applicable.

\section{Funding}

No funding was received. 


\section{Availability of data and materials}

The datasets used and/or analyzed during the present study are available from the corresponding author on reasonable request.

\section{Authors' contributions}

ZL wrote the manuscript and revised it critically for important intellectual content. ZL and MH interpreted and analyzed the patient data. DL and JC designed the study and performed the experiment. HD and JY were responsible for the analysis and discussion of the data. All authors read and approved the final manuscript.

\section{Ethics approval and consent to participate}

The present study was approved by the Ethics Committee of Xinchang People's Hospital. Patients who participated in this research, signed the informed consent and had complete clinical data.

\section{Patient consent for publication}

Not applicable.

\section{Competing interests}

The authors declare that they have no competing interests.

\section{References}

1. Leeyaphan C, Jiamton S, Chanyachailert P, Surawan T and Omcharoen V: Treatment outcomes and loss to follow-up rate of male patients with gonococcal and nongonococcal urethritis who attended the sexually transmitted disease clinic: An 8-year retrospective study. Indian J Sex Transm Dis AIDS 38: 37-42, 2017.

2. Ong JJ, Sarumpaet A, Chow EP, Bradshaw C, Chen M, Read T and Fairley CK: Should female partners of men with non-gonococcal urethritis, negative for Chlamydia trachomatis and Mycoplasma genitalium, be informed and treated? Clinical outcomes from a partner study of heterosexual men with NGU. Sex Transm Dis 44: 126-130, 2017.

3. Pond MJ, Nori AV, Witney AA, Lopeman RC, Butcher PD and Sadiq ST: High prevalence of antibiotic-resistant Mycoplasma genitalium in nongonococcal urethritis: The need for routine testing and the inadequacy of current treatment options. Clin Infect Dis 58: 631-637, 2014.

4. Ito S, Hatazaki K, Shimuta K, Kondo H, Mizutani K, Yasuda M, Nakane K, Tsuchiya T, Yokoi S, Nakano M, et al: Haemophilus influenzae isolated from men with acute urethritis: Its pathogenic roles, responses to antimicrobial chemotherapies, and antimicrobial susceptibilities. Sex Transm Dis 44: 205-210, 2017.

5. Sethi S, Zaman K and Jain N: Mycoplasma genitalium infections: Current treatment options and resistance issues. Infect Drug Resist 10: 283-292, 2017.

6. Kissinger PJ, White S, Manhart LE, Schwebke J, Taylor SN, Mena L, Khosropour CM, Wilcox L, Schmidt N and Martin DH: Azithromycin treatment failure for Chlamydia trachomatis among heterosexual men with nongonococcal urethritis. Sex Transm Dis 43: 599-602, 2016.

7. González-Lizárraga F, Socías SB, Ávila CL, Torres-Bugeau CM, Barbosa LR, Binolfi A, Sepúlveda-Díaz JE, Del-Bel E, Fernandez CO, Papy-Garcia D, et al: Repurposing doxycycline for synucleinopathies: Remodelling of $\alpha$-synuclein oligomers towards non-toxic parallel beta-sheet structured species. Sci Rep 7: 41755, 2017.
8. Stein GE, Mummaw NL and Havlichek DH: A preliminary study of clarithromycin versus doxycycline in the treatment of nongonococcal urethritis and mucopurulent cervicitis. Pharmacotherapy 15: 727-731, 1995.

9. Cunha BA, Baron J and Cunha CB: Similarities and differences between doxycycline and minocycline: Clinical and antimicrobial stewardship considerations. Eur J Clin Microbiol Infect Dis 37: $15-20,2018$

10. Abdolrasouli A and Roushan A: Corynebacterium propinquum associated with acute, nongonococcal urethritis. Sex Transm Dis 40: 829-831, 2013.

11. Khosropour CM, Manhart LE, Colombara DV, Gillespie CW, Lowens MS, Totten PA, Golden MR and Simoni J: Suboptimal adherence to doxycycline and treatment outcomes among men with non-gonococcal urethritis: A prospective cohort study. Sex Transm Infect 90: 3-7, 2014.

12. Manhart LE, Gillespie CW, Lowens MS, Khosropour CM, Colombara DV, Golden MR, Hakhu NR, Thomas KK, Hughes JP, Jensen NL and Totten PA: Standard treatment regimens for nongonococcal urethritis have similar but declining cure rates: A randomized controlled trial. Clin Infect Dis 56: 934-942, 2013.

13. Braune J, Weyer U, Hobusch C, Mauer J, Brüning JC, Bechmann I and Gericke M: IL-6 regulates M2 polarization and local proliferation of adipose tissue macrophages in obesity. J Immunol 198: 2927-2934, 2017.

14. Kinghorn GR: Neisseria gonorrhoeae. Keywords: Neisseria gonorrhoeae, penicillin sensitivity. Sex Transm Infect 54: 112-114, 1978.

15. Kondo H, Ito S, Hatazaki K, Horie K, Nakane K, Mizutani K, Tsuchiya T, Yasuda M, Yokoi S, Nakano M and Deguchi T: GyrA and/or ParC alterations of haemophilus influenzae strains isolated from the urethra of men with acute urethritis. J Infect Chemother 24: 232-235, 2018.

16. Deguchi T: Proposed treatment strategies for non-gonococcal urethritis. Lancet Infect Dis 17: 1121-1122, 2017.

17. Frølund M, Wikström A, Lidbrink P, Abu Al-Soud W, Larsen N, Harder CB, Sørensen SJ, Jensen JS and Ahrens P: The bacterial microbiota in first-void urine from men with and without idiopathic urethritis. PLoS One 13: e0201380, 2018.

18. Aaronson NK, Ahmedzai S, Bergman B, Bullinger M, Cull A, Duez NJ, Filiberti A, Flechtner H, Fleishman SB, de Haes JC, et al: The European organization for research and treatment of cancer QLQ-C30: A quality-of-life instrument for use in international clinical trials in oncology. J Natl Cancer Inst 85: 365-376, 1993

19. Fayers PM, Aaronson NK, Bjordal K, Groenvold M, Curran D and Bottomley A; On behalf of the EORTC Quality of Life Group: The EORTC QLQ-C30 Scoring Manual. 3rd edition. European Organization for Research and Treatment of Cancer, Brussels, 2001.

20. Nenoff P, Manos A, Ehrhard I, Krüger C, Paasch U, Helmbold P and Handrick W: Non-viral sexually transmitted infections-Epidemiology, clinical manifestations, diagnostics and therapy: Part 2: Chlamydia and mycoplasma. Hautarzt 68: 50-58, 2017 (In German).

21. Handsfield $\mathrm{H}$ : Management of herpetic urethritis and female partners of men with nongonococcal urethritis. Sex Transm Dis 44: 131-133, 2017.

22. Bradshaw CS, Tabrizi SN, Read TR, Garland SM, Hopkins CA, Moss LM and Fairley CK: Etiologies of nongonococcal urethritis: Bacteria, viruses, and the association with orogenital exposure. J Infect Dis 193: 336-345, 2006.

23. Jensen JS, Bradshaw CS, Tabrizi SN, Fairley CK and Hamasuna R: Azithromycin treatment failure in Mycoplasma genitaliumpositive patients with nongonococcal urethritis is associated with induced macrolide resistance. Clin Infect Dis 47: 1546-1553, 2008.

24. Ito S, Yasuda M, Seike K, Sugawara T, Tsuchiya T, Yokoi S, Nakano $M$ and Deguchi T: Clinical and microbiological outcomes in treatment of men with non-gonococcal urethritis with a $100-\mathrm{mg}$ twice-daily dose regimen of sitafloxacin. J Infect Chemother 18: 414-418, 2012.

25. Mena LA, Mroczkowski TF, Nsuami M and Martin DH: A randomized comparison of azithromycin and doxycycline for the treatment of Mycoplasma genitalium-positive urethritis in men. Clin Infect Dis 48: 1649-1654, 2009.

26. Salado-Rasmussen K and Jensen JS: Mycoplasma genitalium testing pattern and macrolide resistance: A Danish nationwide retrospective survey. Clin Infect Dis 59: 24-30, 2014. 
27. Yamagoe M, Osada T, Goto M, Nishida S and Nakamura M: Clinical studies on doxycycline in male nongonococcal urethritis. Jpn J Antibiot 38: 3156-3168, 1985 (In Japanese).

28. Maohua L, Hongtao J, Xiuxin W, Yu Z, Tao S and Hua Y: Clinical analysis of 195 cases of non-gonococcal urethritis treated with doxycycline dispersible tablets. Shanghai Med Pharm J 19: 34-42, 2019.

29. Aparicio NJ, Muchinik G, Levalle O, Tropea L, Guitelman A and Grinstein S: The effect of a treatment with doxycycline on semen of asthenozoospermic patients with T-mycoplasma genital infection. Andrologia 12: 521-524, 1980.
30. Totten PA, Jensen NL, Khosopour CM, Gillespie CW, Jensen JS, Kenny GK, Golden MR and Manhart LE: O19.1 Azithromycin and doxycycline resistance profiles of Mycoplasma genitalium and association with treatment outcomes. Sex Transm Infect 89 (Suppl 1): A62, 2013

31. Li B, Hocking JS, Bi P, Bell C and Fairley CK: The efficacy of azithromycin and doxycycline treatment for rectal chlamydial infection: A retrospective cohort study in South Australia. Intern Med J 48: 259-264, 2018. 